\title{
Even More About The Lattice Of Tense Logics
}

\author{
Marcus Kracht * \\ II. Department of Mathematics \\ Arnimallee 3 \\ 1000 Berlin 33 \\ GERMANY
}

February 6, 2008

\begin{abstract}
The present paper is based on [11], where a number of conjectures are made concerning the structure of the lattice $\operatorname{Ext}(\mathbf{K} t)$ of normal extensions of the tense logic $\mathbf{K} t$. That paper was mainly dealing with splittings of $\operatorname{Ext}(\mathbf{K} t)$ and some sublattices, and this is what I will concentrate on here as well. The main tool in analysing the splittings of $\operatorname{Ext}(\mathbf{K} t)$ will be the splitting theorem of [7]. In [11] it was conjectured that each finite subdirectly irreducible algebra splits the lattice of normal extensions of $\mathbf{K} \mathbf{4} t$ and $\mathbf{S} \mathbf{4} t$. We will show that this is not the case and that on the contrary only very few and trivial splittings of the mentioned lattices exist.
\end{abstract}

${ }^{*}$ I wish to thank Prof. Rautenberg for suggesting this work to me and for waiting patiently for two years until I started it. Thanks also to two anonymous referees and Frank Wolter for helpful discussion of this paper. One of the referees deserves special mentioning for his precise and detailed criticism. 


\section{A Splittings in Lattices of Logics}

Definition 1 Let $\mathcal{L}$ be a lattice. $p \in \mathcal{L}$ is said to split $\mathcal{L}$ iff there is an element $q$ such that $\forall x \in \mathcal{L}: x \geq q \Leftrightarrow x \not \leq p$. Equivalently, if $\mathcal{L}$ is complete $p$ splits $\mathcal{L}$ iff $p$ is prime in $\mathcal{L}$, that is, $p \geq \Pi\left\langle x_{i} \mid i \in I\right\rangle$ implies $p \geq x_{i}$ for some $i \in I$. If $p$ splits $\mathcal{L}, q$ is uniquely determined and called the splitting companion of $p$. We write $\mathcal{L} / p$ for $q$. Dually, an element $q$ co-splits $\mathcal{L}$ if there is a splitting element $p$ such that $\mathcal{L} / p=q$.

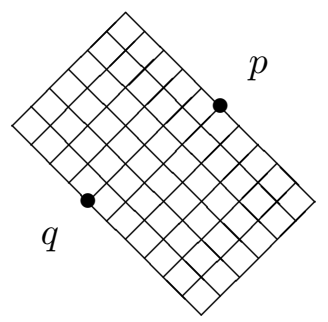

Dually, $p$ co-splits $\mathcal{L}$ iff $p$ is $\sqcup$-prime. Note that if $p$ splits $\mathcal{L}$ then $p$ must be $\Pi$-irreducible, that is, $p=\Pi\left\langle b_{i} \mid i \in I\right\rangle$ implies $p=b_{i}$ for some $i \in I$. In the lattices we are going to study an element $p$ is $\bigsqcup$-prime iff it is $\bigsqcup$-irreducible; however, $П$-irreducibility does not imply $\Pi$-primeness. The definition of a splitting is with minor differences the one given in [8] who also noted that $p$ splits a lattice iff it is prime in that lattice. For a brief history of the notion of a splitting see [7]. The classical case of a splitting is a result by Dedekind which states that a lattice $\mathcal{L}$ is modular iff the lattice $N_{5}$ pictured below cannot be embedded into $\mathcal{L}$.
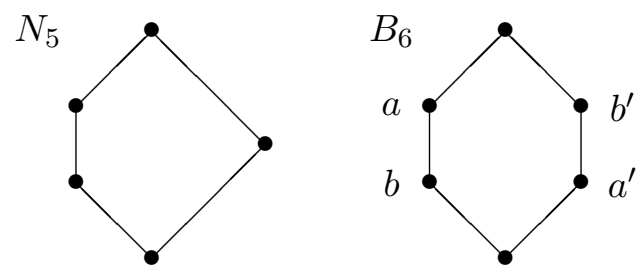

This can be rephrased by saying that an equational theory $\Theta$ of lattices contains the modular laws iff it is not contained in the theory of $N_{5}$; in other words, the equational theory of $N_{5}$ splits the lattice of equational theories of lattices. A less familiar example is a folklore result from the theory of orthomodular lattices (see [6]). An ortholattice $\left\langle L, \sqcap, \sqcup,{ }^{\prime}\right\rangle$ is orthomodular iff it does not contain the ortholattice $B_{6}$ as a sublattice. 
In [2], [3], [4] and [9], [10], [12] splittings have been applied to modal logics and quite interesting theorems were proved about splittings in this particular setting. There are three reasons why splittings should interest a logician. The first was already discussed in [8]. Take two logics $\Theta$ and $\Lambda$ and assume that $\Theta \subseteq \Lambda$. Then define $\nabla_{\Theta}(\Lambda)$ to be the set of cardinalities of independent axiomatizations of $\Lambda$ over $\Theta$. Formally, $n \in \nabla_{\Theta}(\Lambda)$ iff there is a set $X$ of axioms of cardinality $n$ such that for no $Y \subseteq X$ of cardinality less than $n, \Lambda=\Theta(Y)$. In our case, since the language is countable and contains conjunction, there are only three possibilties for $\nabla_{\Theta}(\Lambda)$ :

$$
\begin{aligned}
& \nabla_{\Theta}(\Lambda)=\emptyset \\
& \nabla_{\Theta}(\Lambda)=\{1, \ldots, n\} \\
& \nabla_{\Theta}(\Lambda)=\{\omega\}
\end{aligned}
$$

The first occurs exactly when $\Lambda=\Theta$. In the second case $\Lambda$ is called finitely axiomatizable over $\Theta$. Now McKenzie notes in [8] that

Proposition $2 \Lambda$ co-splits $\operatorname{Ext}(\Theta)$ iff $\nabla_{\Theta}(\Lambda)=\{1\}$.

Since for example $\mathbf{S} 5$ co-splits $\operatorname{Ext}(\mathbf{S} 4)([12])$ any axiomatization of S5 over $\mathbf{S} 4$ must contain a single axiom which axiomatizes $\mathbf{S 5}$ over $\mathbf{S 4}$. $\mathbf{G}$ on the other hand does not split $\operatorname{Ext}(\mathbf{K} 4)$ and consequently, since $1 \in \nabla_{K 4}(\mathbf{G})$, we also have $2 \in \nabla_{K 4}(\mathbf{G})$.

A second property of splittings is their strong connection with completeness questions. Let $F s_{\Theta}(\Lambda)$ denote the set of logics containing $\Theta$ with exactly the same Kripke-models as $\Lambda$. Following [12] we call $F s_{\Theta}(\Lambda)$ the FINE-spectrum of $\Lambda$ over $\Theta . \quad \Lambda$ is called complete if $\Lambda$ is the largest element of $F s_{\Theta}(\Lambda)$ and strictly complete if it is the only element of its spectrum. In [12] and [3] it is shown that if both $\Theta$ and $\Lambda$ are complete and $\Lambda$ is a union of co-splitting logics of $\operatorname{Ext}(\Theta)$ then $\Lambda$ is strictly complete. Since the proofs are given there only for special settings we will prove the general statement here. Let $\mathcal{L}=\langle L, \sqcap, \sqcup\rangle$ be a complete lattice and $N \subseteq L$ and arbitrary subset. Let $S p_{N}(a)=\{b \mid(\forall x \in N)(x \geq a \Leftrightarrow x \geq b)\}$ denote the $\mathbf{N}$-spectrum of $a$ in $\mathcal{L}$. Call $a \mathrm{~N}$-complete if $a$ is the largest element in its N-spectrum and strictly $\mathbf{N}$-complete if it is the only element in its $\mathrm{N}$-spectrum. $a$ is N-complete iff $a=\prod\langle b \mid b \in N, b \geq a\rangle$. In the special case where $N$ is the set $\mathbb{P}$ of prime (or splitting) elements of $\mathcal{L}$ we write $\operatorname{Psp}(a)$ for $\operatorname{Sp}_{\mathbb{P}}(a)$ and call this the prime spectrum of $a$. In contrast to other spectra, the prime spectrum is always an interval.

Proposition 3 For every a there are $a_{0}$ and $a^{0}$ such that $\operatorname{Psp}(a)=\left[a_{0}, a^{0}\right]$. $a^{0}$ is $\mathbb{P}$-complete and $a_{0}$ is a union of co-splitting logics. Consequently, if $a=a_{0}$ and $a$ is $\mathbb{P}$-complete then $a$ is strictly $\mathbb{P}$-complete. 
Proof. Clearly, $a^{0}=\Pi\langle p \mid p \in \mathbb{P}, p \geq a\rangle$ satisfies the requirements. Now define $a_{0}=\bigsqcup\langle\mathcal{L} / p \mid p \in \mathbb{P}, p \nsupseteq a\rangle$. If $c \in \operatorname{Psp}(a)$ then $a_{0} \leq c$, for if $p \in \mathbb{P}$ then $p \nsupseteq a \Leftrightarrow p \nsupseteq c$ whence $\mathcal{L} / p \leq a \Leftrightarrow \mathcal{L} / p \leq c$ and so $c \geq \bigsqcup\langle\mathcal{L} / q \mid p \in \mathbb{P}, p \nsupseteq a\rangle=a_{0}$. Conversely, if $a_{0} \leq c \leq a^{0}$ then $p \geq c \Leftrightarrow p \geq a$ for all $p \in \mathbb{P}$ and so $c \in \operatorname{Psp}(a)$.

It is easily seen that if $N \supseteq \mathbb{P}$ then $\operatorname{Sp}_{N}(a) \subseteq \operatorname{Psp}(a)$. Hence if $a=a_{0}$ and $a$ is $\mathrm{N}$-complete, $a$ is $\mathbb{P}$-complete and thus strictly $\mathbb{P}$-complete and therefore strictly N-complete. To connect this result with modal logic, consider again the case of $\mathbf{S 5}$. It is known that $\Lambda$ splits the lattice of $\mathbf{S} 4$-extensions iff $\Lambda$ is the logic of a finite one-generated Kripke frame. Thus $\mathbb{P}$ is the set of logics $L \Gamma$ for finite one-generated $\Gamma$ and $N$ the set of $L \Gamma$ for arbitrary Kripke-frames $\Gamma$. S5 is a co-splitting logic, thus $\mathbf{S} 5=\mathbf{S} 5_{0}$ and $\mathbf{S} 5$ is complete. Hence S5 is strictly complete. Moreover, our proposition gives us something stronger; namely, since $\mathbf{S 5}$ has the finite model property (f.m.p.), S5 is $\mathbb{P}$-complete. Hence any extension of $\mathbf{S} \mathbf{4}$ admitting the same finite one-generated frames as $\mathbf{S 5}$ is identical to $\mathbf{S 5}$. For example, this allows to decide positively that $\mathbf{S} 4.5=\mathbf{S} 4(\square \diamond \square p \rightarrow \square p)=\mathbf{S 5}$. Moreover, any extension of S5 lacks at least one of the finite models of S5. This allows to conclude quite quickly that the lattice of extensions of $\mathbf{S 5}$ is isomorphic to $1+\omega^{\star}$, where $\omega^{\star}$ is the converse of $\omega$ and $n \in \omega^{\star}$ represents the logic of the $n$-point cluster.

Finally, splittings relate quite intimately with certain decidability questions. For suppose that $\Theta$ splits $\operatorname{Ext}(\Lambda)$; then the problem ' $\Lambda(P) \supseteq \Lambda / \Theta$ ' is equivalent to ' $P \notin \Theta$ '; thus if $\Theta$ is decidable, so is the problem ' $\Lambda(P) \supseteq \Lambda / \Theta$ '. If in addition $\Lambda / \Theta$ is decidable, the problem ' $\Lambda(P) \subseteq \Lambda / \Theta$ ' is decidable as well. This can be generalized to the following proposition.

Proposition 4 Let $\Theta_{i}, i \in n$, be logics splitting the lattice $\operatorname{Ext}(\Lambda)$. If all $\Theta_{i}$ are decidable, e.g. if they are modal theories of some finite frames, and if also $\bigsqcup\left\langle\Lambda / \Theta_{i} \mid i \in n\right\rangle$ is decidable, then the problem ' $\Lambda(P)=\bigsqcup\left\langle\Lambda / \Theta_{i} \mid i \in n\right\rangle$ ' is decidable as well.

Proof. Observe that ' $\Lambda(P) \supseteq \sqcup\left\langle\Lambda / \Theta_{i} \mid i \in n\right\rangle$ ' is equivalent to ' $P \notin \Theta_{0} \& P \notin$ $\Theta_{1} \& \ldots \& P \notin \Theta_{n-1}$ '.

It seems therefore a natural question whether there are interesting applications of splittings in tense logics. Since the extension lattices of K4 and $\mathbf{S} \mathbf{4}$ are rather rich in splittings it might be hoped that the extension lattices of their tense extensions $\mathbf{K} 4 t$ and $\mathbf{S} 4 t$ defined below share this property. Our results, however, are negative. In tense-logics there are very few splittings. Thus there are few logics which share the rather pleasing properties of co-splitting extension. And this in turn suggests that in general modal logic the applications of this method will be rather limited. 


\section{B Tense Logics}

The lattices we will discuss will be the extension lattices $\operatorname{Ext}(\Theta)$ for some normal tense logic $\Theta$. As usual, there are denumerably many propositional variables $p, q, r, \ldots$, the boolean connectives $\wedge, \neg, \rightarrow, \ldots$ and the two modal operators $\square^{+}, \square^{-}$with their duals $\diamond^{+}, \diamond^{-}$. A normal tense-logic is a set $\Lambda$ of wff's composed from variables and the above symbols which contains all the axioms of classical logic, the axioms $\mathrm{BD}^{+}: \square^{+}(p \rightarrow q) \rightarrow .^{+} p \rightarrow \square^{+} q$, $\mathrm{BD}^{-}: \square^{-}(p \rightarrow q) \rightarrow .^{-} p \rightarrow \square^{-} q, \mathrm{Z}^{+}: p \rightarrow \square^{+} \diamond^{-} p, \mathrm{Z}^{-}: p \rightarrow \square^{-} \diamond^{+} p$ and is closed under MP, substitution and $\mathrm{MN}^{+}: P / \square^{+} P, \mathrm{MN}^{-}: P / \square^{-} P$. The smallest tense logic is denoted by $\mathbf{K} t$. The lattice $\operatorname{Ext}(\mathbf{K})$ of normal modal logics can be embedded into the lattice of normal tense logics by interpreting $\square$ as $\square^{+}$which turns the language of modal logics into a sublanguage of tense logic. This defines a translation $(-)^{+}$of modal formulae into tense formulae but it can also be used to translate modal logics into tense logics. If $\Lambda \in$ $\operatorname{Ext}(\mathbf{K})$ then take $\Lambda^{+}$to be the smallest normal tense logic containing all $P^{+}$ with $P \in \Lambda$. This defines a $\sqcup$-homomorphism $(-)^{+}: \operatorname{Ext}(\mathbf{K}) \rightarrow \operatorname{Ext}(\mathbf{K} t)$. Instead of $\Lambda^{+}$we usually write $\Lambda t$, following common practice. Likewise, the map $(-)^{-}$is defined by identifying $\square$ with $\square^{-}$. Thus, Alt $_{1}^{+}$denotes the formula resulting from the axiom Alt $_{1}$ by replacing $\square$ by $\square^{+}$throughout and $\mathbf{A l t}_{1}^{-}$the formula resulting from $\mathbf{A l t}_{1}$ by replacing $\square$ uniformly by $\square^{-}$. So, $\mathbf{K} 4 t$ is the same as $\mathbf{K} \mathbf{4}^{+}$(and incidentally also the same as $\mathbf{K} \mathbf{4}^{-}$). Equally, $\mathbf{K 4 . 1} t=\mathbf{K} \mathbf{4}^{+} . \mathbf{1}^{+}\left(\neq \mathbf{K} \mathbf{4}^{-} . \mathbf{1}^{-}\right)$!. There are also maps $\left.(-)_{+},(-)\right)_{-}$: $\operatorname{Ext}(\mathbf{K} t) \rightarrow \operatorname{Ext}(\mathbf{K})$ defined by restricting a tense logic $\Lambda$ to its fragments without $\square^{-}$vz. without $\square^{+}$. Both are $\Pi$-homomorphisms and by semantic inspection $\Lambda=\left(\Lambda^{+}\right)_{+}=\left(\Lambda^{-}\right)_{-}$. Moreover, $(-)_{+}$is the right adjoint of $(-)^{+}$, that is, for all $\Lambda, \Theta: \Lambda \subseteq \Theta_{+} \Leftrightarrow \Lambda^{+} \subseteq \Theta$; and dually for $(-)_{-}$and $(-)^{-}$.

The semantics for tense logics is straightforward from the simple modal case. A tense algebra is an object $\mathfrak{A}:=\left\langle\mathbf{A}, \tau^{+}, \tau^{-}\right\rangle$, where $\mathbf{A}=\langle A,-, \cap, 1\rangle$ is a boolean algebra and $\tau^{+}, \tau^{-}$unary operators satisfying $\tau^{\circ} 1=1$ and $\tau^{\circ}(a \cap b)=\tau^{\circ} a \cap \tau^{\circ} b, \circ=+,-$. A tense-frame is an object $\Gamma=\langle G, \triangleleft\rangle$, where $G$ is a set and $\triangleleft \subseteq G \times G$ a relation on $G$ i.e. tense-frames are just Kripke-frames. A map $p: \Gamma \rightarrow \Delta, \Delta=\langle D, \triangleleft\rangle$, is a t-morphism iff

$(\triangleleft h) \quad x \triangleleft_{\Gamma} y \Rightarrow p(x) \triangleleft_{\Delta} p(y)$

$(+c) \quad p(x) \triangleleft_{\Delta} y \Rightarrow(\exists u)\left(p(u)=y \& x \triangleleft_{\Gamma} u\right)$

$(-c) \quad y \triangleleft_{\Delta} p(x) \Rightarrow(\exists u)\left(p(u)=y \& u \triangleleft_{\Gamma} x\right)$

If $p$ is injective, we say that $\Gamma$ is a generated subframe of $\Delta$. It is easy to see that generated subframes correspond to full connected subsets of $\Delta$. Hence, if $\Delta$ is connected and $\Gamma$ a generated subframe, it follows that $\Gamma=\Delta$. 
If $S \subseteq D$ is a subset, we call the least generated subframe containing $S$ the transit of $S$ in $\Delta$ and denote it by $\mathbb{T}(S, \Delta)$. We say, $\Delta$ is one-generated if $\Delta=\mathbb{T}(\{s\}, \Delta)$ for some (and hence any) $s \in D$. Evidently, $\Delta$ is onegenerated iff $\Delta$ is connected. We write $p: \Gamma \rightarrow \Delta$ whenever $p$ is a t-morphism and $p: \Gamma \leadsto \Delta$ for an arbitrary map.

There is a dual correspondence between finite frames and finite algebras. A finite algebra $\mathfrak{A}$ uniquely determines a frame $F(\mathfrak{A})=\left\langle G(\mathfrak{A}), \triangleleft_{\mathfrak{A}}\right\rangle$ with $G(\mathfrak{A})$ the set of atoms of $A$ and $a \triangleleft_{\mathfrak{A}} b$ iff $a \leq-\tau^{+}-b$. A finite frame $\Gamma=\langle G, \triangleleft\rangle$ uniquely determines an algebra $A(\Gamma)=\left\langle\wp(G), \tau_{\Gamma}^{+}, \tau_{\Gamma}^{-}\right\rangle$where $\wp(G)$ is the powerset algebra of $G$ and $\tau_{\Gamma}^{+} A=\{g \in G \mid \forall h: g \triangleleft h \Rightarrow h \in A\}$, $\tau_{\Gamma}^{-} A=\{g \in G \mid \forall h: h \triangleleft g \Rightarrow h \in A\}$. It is standard to verify $\Gamma \cong F(A(\Gamma))$ and $\mathfrak{A} \cong A(F(\mathfrak{A}))$.

Proposition $\mathbf{5}$ For finite $\mathfrak{A}$ the following are equivalent:

(i) $\mathfrak{A}$ is subdirect irreducible.

(ii) $\mathfrak{A}$ is directly irreducible.

(iii) $F(\mathfrak{A})$ is connected.

(iv) $\mathfrak{A}$ is simple.

If either of (i) - (iv) holds, then LA is П-irreducible.

Proof. If (i) holds then (ii) holds as well. Moreover, since $F(\mathfrak{A} \times \mathfrak{B})$ is the disjoint union (= co-product) of the frames $F(\mathfrak{A})$ and $F(\mathfrak{B})$, (ii) and (iii) are clearly equivalent. Finally, if $F(\mathfrak{A})$ is connected, it is one-generated and therefore $\mathfrak{A}$ subdirectly irreducible (Goldblatt [89]) whence (iii) implies (i). By duality, homomorphic images of $\mathfrak{A}$ are generated subsets of $F(\mathfrak{A})$ and thus (iii) and (iv) are equivalent. Finally, for finite $\mathfrak{A}$ the implication (i) $\Rightarrow$ (v) follows from universal algebra.

For infinite algebras some of the equivalences are not valid. Finally, recall the notion of a generalized frame from [13] which is a pair $\langle\Gamma, \mathbb{G}\rangle$ such that $\Gamma$ is a tense-frame and $\mathbb{G} \subseteq 2^{\Gamma}$ a set closed under complementation, intersection and the operators $\tau_{\Gamma}^{+}, \tau_{\Gamma}^{-}$. We will not use them much; let us just state that every tense algebra can be thought of a set algebra over a tense-frame whose points are the ultrafilters of that algebra. If $\Gamma$ is a frame and $X$ is a set of variables, $\gamma: X \rightarrow 2^{G}$ is called a valuation. If $P$ is based on variables of $X,\langle\Gamma, \gamma, g\rangle \models P$ is well defined by induction on $P$, the critical cases being $\langle\Gamma, \gamma, g\rangle \models \square^{+} Q$ iff $\forall h: g \triangleleft h \Rightarrow\langle\Gamma, \gamma, h\rangle \models Q$ and $\langle\Gamma, \gamma, g\rangle \models \square^{-} Q$ iff $\forall h: h \triangleleft g \Rightarrow\langle\Gamma, \gamma, h\rangle \models Q$. We write $\langle\Gamma, \gamma\rangle \models Q$ if $\forall g \in G:\langle\Gamma, \gamma, g\rangle \models Q$ and $\Gamma \models Q$ if $\forall \gamma: \operatorname{var}(Q) \rightarrow 2^{G}:\langle\Gamma, \gamma\rangle \models Q$. The logic of $\Gamma$ is simply $L \Gamma=\{Q \mid \Gamma \models Q\}$. A logic $\Lambda$ is called tabular iff there is a finite $\Gamma$ such that $\Lambda=L \Gamma$. Analogously, a valuation $\alpha: X \rightarrow A$ into an algebra $\mathfrak{A}=\left\langle\mathbf{A}, \tau^{+}, \tau^{-}\right\rangle$is defined. 
If $\Gamma$ is finite, one can define a diagram of $\Gamma$ on a set $\left\{p_{g} \mid g \in G\right\}$ of propositional variables as follows:

$$
\begin{aligned}
\Delta(\Gamma)= & \bigwedge\left\langle p_{s} \rightarrow \neg p_{t} \mid s \neq t\right\rangle \\
& \wedge \bigwedge\left\langle p_{s} \rightarrow \diamond^{+} p_{t} \mid s \triangleleft t\right\rangle \\
& \wedge \bigwedge\left\langle p_{s} \rightarrow \square^{+} \neg p_{t} \mid s \rtimes t\right\rangle \\
& \wedge \bigvee\left\langle p_{s} \mid s \in G\right\rangle
\end{aligned}
$$

Here, $s$ and $t$ range over points of $G$. With the abbreviation $\square^{(0)} P:=P$ and $\square^{(n+1)} P:=\square^{+} \square^{(n)} P \wedge \square^{-} \square^{(n)} P \wedge \square^{(n)} P$ we can now state the

Theorem 6 (Splitting Theorem) Let $\Theta$ be a tense-logic with f.m.p. Then $\Lambda$ splits $\operatorname{Ext}(\Theta)$ iff $\Lambda=L \Gamma$, where $\Gamma$ is finite (and thus has a diagram $\Delta(\Gamma)$ ), connected and there is a $m$ such that for every $\Theta$-algebra $\mathfrak{A}$ :

(†) If $\mathfrak{A} \not \models \square^{(m)} \Delta(\Gamma) \rightarrow \neg p_{g}$ then for every $m^{\prime} \mathfrak{A} \not \models \square^{\left(m^{\prime}\right)} \Delta(\Gamma) \rightarrow \neg p_{g}$.

A proof of this splitting theorem can be found in [7]. Suffice it to say here that if $\Lambda$ splits $\operatorname{Ext}(\Theta)$ then $\Lambda$ is $\Pi$-irreducible and so $\Lambda=L \mathfrak{A}$ for a s.i. $\mathfrak{A}$. So if $\Theta$ has f.m.p., then $\Theta=\Pi\langle L \mathfrak{A}| \mathfrak{A}$ f.s.i. $\rangle=\Pi\langle L \Gamma| \Gamma$ finite, connected $\rangle$. And if $\mathfrak{B}$ is infinite or not s.i. then for no finite connected $\Gamma L \Gamma \subseteq L \mathfrak{B}$ but $\Pi\langle L \Gamma| \Gamma$ finite,connected $\rangle \subseteq L \mathfrak{B}$, so that $L \mathfrak{B}$ is not prime in $\operatorname{Ext}(\Theta)$. Since for any $\Lambda$ there is a $\mathfrak{B}$ such that $L \mathfrak{B}=\Lambda, \Lambda$ splits $\operatorname{Ext}(\Theta)$ iff $\Lambda$ is the logic of a one-generated finite frame. If we call a logic $\Theta \mathbf{k}$-transitive if $\square^{(k)} p \rightarrow \square^{(k+1)} p \in \Theta$ we have the following corollary essentially due to Rautenberg ([9]):

Corollary 7 Let $\Theta$ be a k-transitive tense logic with f.m.p. Then $\Lambda$ splits $\operatorname{Ext}(\Theta)$ iff $\Lambda=L \Gamma$ for a finite and connected $\Gamma$.

The intuition behind k-transitivity is the following: call a function $w$ : $\{0, \ldots, \ell\} \rightarrow G, \ell \in \omega$, a path of length $\ell$ in $\Gamma$, if $\forall i<\ell: w(i) \triangleleft w(i+$ 1) or $w(i) \triangleright w(i+1)$. A frame is k-transitive if any pair of points that can be connected at all can be connected by a path of length $\leq k$. The axiom of k-transitivity forces all frames to be k-transitive. k-transitive logics are therefore easy to deal with. But the logics $\mathbf{K 4} t$, $\mathbf{S} 4 t$, which are of considerable interest, are not among them although the frames for those logics are transitive as modal frames, which may have caused the error in [11] in which he states that $\mathbf{S} 4 t$ and $\mathbf{K} 4 t$ are 1-transitive. They are not, and this forces us to look more deeply into the structure of their extension lattices.

In [7], a generalization of a theorem by Blok in [3] to poly-modal logics is proved. Call $\Gamma$ cycle-free if there is a $k \in \omega$ such that there exists no 
path of length $k$ in $\Gamma$. Then for any cycle-free tense frame $\Gamma L \Gamma$ splits $\mathcal{E} \Theta$ as was conjectured in [11]. However, it is quickly checked that there is exactly one cycle-free tense frame namely $\langle\{s\}, \emptyset\rangle$, which we denote by $\mathbf{x}$.

\section{Subreducing Sequences}

To prove that a logic $\Lambda$ does not split the lattice of extensions of $\operatorname{Ext}(\Theta)$ is an altogether different task than to prove that it does. The most direct way is to show that $\Lambda$ is not prime in the lattice by naming a sequence of logics $\left\langle\Xi_{i} \mid i \in \omega\right\rangle$ such that $\Lambda \nsupseteq \Xi_{i}$ for any $i \in \omega$ but $\Theta \supseteq \bigcap\left\langle\Xi_{i} \mid i \in \omega\right\rangle$. This motivates the following

Definition 8 A sequence $\left\langle a_{i} \mid i \in I\right\rangle$ of elements of a complete lattice $\mathcal{L}$ subreduces $p$ if for all $i \in I: p \nsupseteq a_{i}$ but $p \geq \Pi\left\langle a_{i} \mid i \in I\right\rangle$. A sequence of frames $\left\langle\Delta_{i} \mid i \in \omega\right\rangle$ subreduces $\Gamma$ if $\left\langle L \Delta_{i} \mid i \in \omega\right\rangle$ subreduces $L \Gamma$.

A simple example for a subreducing sequence is the sequence of unravellings or ramification stages for a modal frame with a cycle $([10])$. It can be shown that a modal logic $\Lambda$ splits the lattice $\operatorname{Ext}(\mathbf{K})$ only if $\Lambda=L \Gamma$ for a finite, one-generated and cycle-free $\Gamma$ by showing that if $\Gamma$ contains a cycle, the infinite series of ramification stages subreduces $\Gamma$. Although of some importance, the ramification technique is not general enough for our purpose. Even $\mathbf{K} t$ requires a more sophisticated technique which I will describe now. The essence is the notion of a local t-morphism. Before we can define it let us introduce some more technology. Define the modal degree $d g(P)$ of $P$ inductively by

$$
\begin{array}{lll}
d g(p) & =0 & p \text { a variable } \\
d g(\neg P) & =d g(P) & \\
d g(P \wedge Q) & =\max \{d g(P), d g(Q)\} & \\
d g\left(\square^{+} P\right) & =d g(P)+1 & \\
d g\left(\square^{-} P\right) & =d g(P)+1 &
\end{array}
$$

Write $L^{n}(\Gamma)$ for the set of formulae $P$ of degree $\leq n$ such that $\Gamma \models P$. Then by the Compactness Theorem $\left\langle\Delta_{i} \mid i \in \omega\right\rangle$ subreduces $\Gamma$ if $L^{i}\left(\Delta_{i}\right) \subseteq L^{i}(\Gamma)$ but $L \Delta_{i} \nsubseteq L \Gamma$ for all $i \in \omega$. Alternatively, if $U$ is a non-principal ultrafilter on $\omega$ then $L\left(\prod_{U} \Delta_{i}\right) \subseteq L \Gamma$ and hence $\bigcap\left\langle L \Delta_{i} \mid i \in \omega\right\rangle \subseteq L \Gamma$. Now if $\Gamma$ is a frame and $g \in G$, define $T^{k}(g, \Gamma)$ to be the set of points $s$ such that there is a path of length $\leq k$ from $g$ to $s$ and let $s \triangleleft t$ iff $s \triangleleft_{\Gamma} t$. This defines the $k$ transit $\mathbb{T}^{k}(g, \Gamma)=\left\langle T^{k}(g, \Gamma), \triangleleft\right\rangle$ of $g$ in $\Gamma$. Now let $p: \Delta \sim \Gamma$ be an arbitrary 
map. We call $p$ a $k$--localic t-morphism with respect to $g$ iff $p$ satisfies $(\triangleleft h)$ and $( \pm c)$ only locally, that is, for all $x \in \mathbb{T}^{k-1}(g, \Gamma), y \in \mathbb{T}^{k}(g, \Gamma)$

$(\triangleleft h) x \triangleleft y \Rightarrow p(x) \triangleleft p(y)$

$(+c) p(x) \triangleleft y \Rightarrow \exists u(p(u)=y \& x \triangleleft u)$

$(-c) y \triangleleft p(x) \Rightarrow \exists u(p(u)=y \& u \triangleleft x)$

The main reason for studying localic t-morphisms is the following lemma, which makes use of the notion of an admissible map. A map $\pi: \Delta \leadsto \Gamma$ is called admissible for $\gamma: X \rightarrow 2^{G}$ if all sets $\gamma(p), p \in X$, are unions of fibres of $\pi$, that is, unions of sets of the form $\pi^{-1}(x)$ for $x \in D$. In that case $\gamma$ defines a unique valuation $\delta: X \rightarrow 2^{D}$ via $\delta(p)=\pi[\gamma(p)]$.

Lemma 9 Let $p: \Delta \leadsto \Gamma$ be k-localic with respect to $g$. Let $\gamma, \delta$ be valuations on $\Gamma, \Delta$ such that $p$ is admissible for $\delta$. Then for all $P$ with $d g(P) \leq k$

$$
\langle\Delta, \delta, g\rangle \models P \Leftrightarrow\langle\Gamma, \gamma, p(g)\rangle \models P
$$

Proof. By induction on $P$.

Now suppose $p: \Delta \leadsto \Gamma$ is $m$-localic with respect to $g \in G$ and that $\Gamma=\mathbb{T}^{k}(g, \Gamma)$. Then $L^{m-k}(\Delta) \subseteq L^{m-k}(\Gamma)$. This is so because if $y \in T^{k}(g, \Gamma)$ then $T^{m-k}(y, \Gamma) \subseteq T^{m}(g, \Gamma)$ and if $P$ is of degree $\leq m-k$ and $\langle\Gamma, \gamma, y\rangle \models$ $P$ then with $\delta$ defined by $\delta(q)=p^{-1}[\gamma(q)]$ we have $\left\langle\Delta, \delta, p^{-1}(y)\right\rangle \models P$ whence $\neg P \notin L^{m-k}(\Gamma)$ implies $\neg P \notin L^{m-k}(\Delta)$. Thus if $\left\langle\Delta_{i} \mid i \in \omega\right\rangle$ is a sequence of frames such that there are points $d_{i} \in D_{i}$ and $p_{i}: \Delta_{i} \leadsto \Gamma$ which are $m$-localic with respect to $d_{i}$ but no $t$-morphism exists from $\Delta_{i}$ to $\Gamma$ then $\left\langle\Delta_{i} \mid i \in \omega\right\rangle$ subreduces $\Gamma$. This connects with the Splitting Theorem as follows. We have $\Gamma \not \models \square^{(m)} \Delta(\Gamma) \rightarrow \neg p_{g}$ for all $m$. Hence $\Delta_{m+k+1} \not \models$ $\square^{(m)} \Delta(\Gamma) \rightarrow \neg p_{g}$ as $d g\left(\square^{(m)} \Delta(\Gamma) \rightarrow \neg p_{g}\right)=m+1$ and $L^{m+1}\left(\Delta_{m+k+1}\right) \subseteq$ $L^{m+1}(\Gamma)$. But if we had $\Delta_{m+k+1} \not \models \square^{(n)} \Delta(\Gamma) \rightarrow \neg p_{g}$ for every $n$, then we take $n$ large so that $\Delta_{m+k+1}=\mathbb{T}^{n}\left(d, \Delta_{m+k+1}\right)$ for some $d$ (assuming that the frames are connected). Then $\Delta_{m+k+1} \not \models \square^{(n)} \Delta(\Gamma) \rightarrow \neg p_{g}$ simply means that $\Delta_{m+k+1} \rightarrow \Gamma$ (see [7]). But we have excluded that. Thus, (†) fails for every $m$ and so $\Gamma$ does not split.

For the definition of subreducing frames we use the following construction. Take two frames $\Gamma, \Delta$ and let $s \in G, t \in D$. Then let $\Gamma^{s} \imath_{t} \Delta$ denote the frame $\left\langle G^{s} \chi_{t} D, \triangleleft\right\rangle$ where $G^{s}{ }_{t} D=G \times\{0\} \cup D \times\{1\}-\{\langle t, 1\rangle\}$ and $\triangleleft$ is defined by

(i) $\quad\langle a, 0\rangle \triangleleft\langle b, 0\rangle$ iff $a \triangleleft_{\Gamma} b$

(ii) $\langle a, 1\rangle \triangleleft\langle b, 1\rangle$ iff $a \triangleleft \Delta b$

(iii) $\langle s, 0\rangle \triangleleft\langle b, 1\rangle$ iff $t \triangleleft_{\Delta} b$

This is well-defined whenever $s \triangleleft s \Leftrightarrow t \triangleleft t$. If $\Gamma, \Delta$ are transitive $\triangleleft$ will be taken to be the transitive closure of the relation defined above. We call 
$\Gamma^{s} \imath_{t} \Delta$ a book and $\Gamma$ and $\Delta$ the pages. When the choice of the points is clear we write $\Gamma$ ? $\Delta$ instead of $\Gamma^{s} \imath_{t} \Delta$. With two points $g \triangleleft h \in G$ fixed we define ${ }^{n} \Gamma, n \geq 1$, by

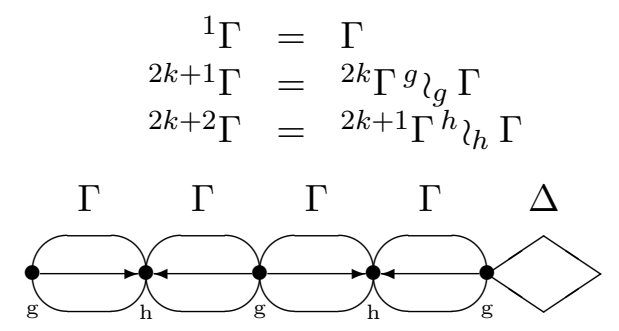

We distinguish the elements of different pages in ${ }^{n} \Gamma$ by indices $0, \ldots, n-1$. The map $\phi: x_{i} \mapsto x$ is a t-morphism; for if $x_{i} \triangleleft y_{j}$ then either $i=j$ and thus $x \triangleleft y$ by (i) and (ii) or $i+1=j$ and $x \triangleleft y$ by (iii). Now if $\phi x_{i} \triangleleft y$ we have $y=\phi y_{i}$ and $x_{i} \triangleleft y_{i}$. Likewise for $x \triangleleft \phi y_{i}$. The same can be shown in the transitive case. By this we see that any map $\psi:{ }^{n} \Gamma \imath \Delta \leadsto \Gamma$ satisfying $\psi\left(x_{i}\right)=x$ is $m-1$-localic with respect to any point $x_{0}$ of the first page. This suggests that by taking a suitable $\Delta$ so that there is no t-morphism from ${ }^{n} \Gamma \imath \Delta$ to $\Gamma$ for any $n \in \omega$ we have a subreducing sequence for $\Gamma$.

\section{Splittings of Tense Logics}

We will study the extension lattices of $\mathbf{K} t, \mathbf{K} 4 t$ and $\mathbf{S} 4 t$. The method is uniform in all three cases and can be transferred to numerous other cases. Each of these logics has f.m.p. (see [5]) and therefore only finite algebras can induce splittings. Thus we can concentrate on the Kripke-frames of those algebras. Let me first introduce you to an important collection of frames, the garlands. A garland is a zigzag frame which looks like this:

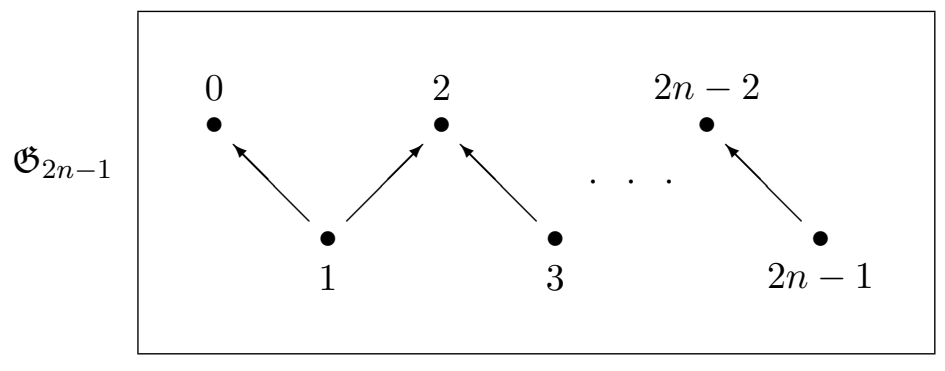

Formally, we define $\mathfrak{G}_{n}$ as a frame $\langle n+1, \triangleleft\rangle$ where $i \triangleleft j$ iff $i=j$ or $i$ is odd and $j=i \pm 1$. Thus, $\mathfrak{G}_{n}$ has $n$ arrows and $n+1$ points. Note that a 
garland is isomorphic to a book where each page is the frame

Garlands can be characterized modally. To see this, recall some notions from standard modal logic. A tense-frame $\Gamma$ is called a cluster if $\Gamma=\langle\{s\}, \emptyset\rangle$ or $\Gamma=\langle G, G \times G\rangle$. A frame is of alternativity $n$ iff every point sees at most $n$ points and can only be seen by at most $n$ incomparable points and it is of depth $\leq n$ if every strictly ascending chain of points has at most $n$ members. Finally, $\Gamma=\langle G, \triangleleft\rangle$ is called meager if there are no two points $s \triangleleft t \triangleleft s$. A connected frame $\Gamma$ is a garland iff it is reflexive, meager, of alternativity 3 and of depth 2. Thus $\Gamma$ is a garland iff $L \Gamma \supseteq \mathbf{G a}:=\mathbf{S} 4 t . \mathbf{G r z}^{+} . \mathbf{A l t}_{3}^{+} \cdot \mathbf{A l t}_{3}^{-} . \mathbf{J}_{2}^{+}$, which is the logic of all garlands. (Recall that frames for $\mathbf{S} 4 t . \mathbf{G r z}{ }^{+}$are meager, that frames for $\mathbf{K} t \cdot \mathbf{A} \mathbf{l t}_{3}^{+} \cdot \mathbf{A} \mathbf{A l}_{3}^{-}$are of alternativity $\leq 3$ and that frames for $\mathbf{J}_{2}^{+}$are of depth 2.) We first prove an important

Lemma 10 Suppose that $\Gamma$ is not a cluster. Then $L \Gamma$ splits $\operatorname{Ext}(\boldsymbol{K} t)$, $\operatorname{Ext}(\boldsymbol{K} 4 t)$ and $\operatorname{Ext}(\boldsymbol{S} 4 t)$ only if $\Gamma$ is a garland.

Proof. $\Gamma$ is finite and connected and $\sharp G=n$. Since $\Gamma$ is not a cluster, there are points $g, h$ such that $g \triangleleft h \Varangle g$. Then $\Gamma=\mathbb{T}^{n}(g, \Gamma)$. Now consider the frame ${ }^{m} \Gamma \imath_{0} \mathfrak{G}_{2 n+8}$. This is well defined in case $g$ and $h$ are both reflexive points. In case one of them is not reflexive we take ${ }^{m} \Gamma \imath_{0} \mathfrak{G}_{2 n+8}^{\circ}$ instead, where $\mathfrak{G}_{2 n+8}^{\circ}$ is identical to $\mathfrak{G}_{2 n+8}$ except that $0 \nless 0$. We have to show that if there is a t-morphism $p:{ }^{m} \Gamma \imath_{0} \mathfrak{G}_{2 n+8}^{(\circ)} \rightarrow \Gamma$ then (a) $\Gamma$ is reflexive and transitive, (b) $\Gamma$ is of alternativity $\leq 3$, (c) $\Gamma$ is meager and (d) $\Gamma$ is of depth $\leq 2$. For then $\Gamma$ splits the lattice only if it satisfies (a) - (d) and thus is a garland. The proof goes as follows. Consider $g=p(n+4)$ where $n+4 \in{ }^{m} \Gamma 2_{0} \mathfrak{G}_{2 n+8}^{(\circ)}$ is in the last page i.e. is right in the middle of the garland. Now as $\Gamma=\mathbb{T}^{n}(g, \Gamma)$ any point $t \in G$ can be connected by a path $v$ of length $\leq n$ from $g$. By $( \pm c)$ this path $v$ has a preimage $w$ with starting point $n+4$. The end point $e$ of $w$ must be a point of the garland, moreover, it lies in between 4 and $2 n+4$. This implies (a) that $e \triangleleft e$. Consequently, $t \triangleleft t$ since $t=p(e)$. And if $t \triangleleft s \triangleleft r$ then by $(+c)$ there are preimages $x, y$ with $e \triangleleft x \triangleleft y$. But as the garlands are transitive, $e \triangleleft y$ and so $t \triangleleft r$. To show (b), $t$ has at most three successors one of which is $t$, since each successor has a preimage succeeding $e$. But $e$ has at most three successors in ${ }^{m} \Gamma \imath_{0} \mathfrak{G}_{2 n+8}^{(\circ)}$. Likewise for predecessors of $t$. Now, suppose that $t \triangleleft u \triangleleft t$. Then by $(+c)$ there is a $x$ such that $e \triangleleft x, p(x)=u$ and a $y$ such that $x \triangleleft y$ and $p(y)=e$. Now either $e=x$ or $x=y$. If $e \neq x$ then $y=x$; thus $u=p(x)=p(e)=t$. If $x=e$ then also $u=t$. This shows (c). For (d) observe similarly that if we had an ascending chain $t \triangleleft u \triangleleft v$ then we could find an ascending chain $e \triangleleft f \triangleleft h$ such that $p(f)=u, p(v)=h$; but then either $e=f$ or $f=h$, so 
that this chain is not strictly ascending. Neither is then $t \triangleleft u \triangleleft v$ strictly ascending.

This considerably reduces the class of possible splitting frames. However, we will also show that most of the garlands and clusters cannot split any of these logics. This we do by establishing a lemma on splittings of $\operatorname{Ext}(\mathbf{G a})$.

Lemma $11 L \Gamma_{n}$ splits $\operatorname{Ext}(\boldsymbol{G a})$ iff $n \leq 1$.

Once this lemma is proved it follows that $\Gamma_{n}$ cannot split $\operatorname{Ext}(\mathbf{K} t), \operatorname{Ext}(\mathbf{K} 4 t)$ nor $\operatorname{Ext}(\mathbf{S} 4 t)$ for $n>1$ since all these lattices contain $\operatorname{Ext}(\mathbf{G a})$.

Proposition 12 There is a t-morphism $p: \mathfrak{G}_{m} \rightarrow \mathfrak{G}_{n}$ iff $n=0, m=\omega$ or $n$ divides $m$.

Proof. $(\Rightarrow)$ Suppose $p: \mathfrak{G}_{m} \rightarrow \mathfrak{G}_{n}$ is a t-morphism. It is clear that $n=0$ is always possible. Thus let $n>0$. Write $i \equiv j$ for $p(i)=p(j)$. We now have the following

Claim 13 On the condition that $n>0$, if $i \equiv j$ then $i \equiv j(\bmod 2)$. Moreover, if $i \equiv j$ then $i-1 \equiv j-1$ or $\equiv j+1$ and $i+1 \equiv j-1$ or $\equiv j+1$, whenever these points exist.

For suppose $i \equiv j$ and that $i$ is even and $j$ is odd. Then $j \triangleleft j-1, j$ and if $j+1 \leq m$ then also $j \triangleleft j+1$. By $(+c)$ and the fact that $i \triangleleft k$ iff $k=i$ we get $j-1 \equiv i$ and $j+1 \equiv i$. Similarly, if $i>0$ then by $(-c) i-1 \equiv j$ and if $i<m$ also $i+1 \equiv j$. Continuing this argument we get $k \equiv \ell$ for all $k, \ell$ and hence $n=0$, which we have excluded. Now let again $i \equiv j$. Then if both are even, $i-1, i, i+1 \triangleleft i$ and $j-1, j, j+1 \triangleleft j$ whenever these points exist. By $(-c), i-1 \equiv j-1, j$ or $j+1$. But since $j$ is even and $i-1$ is odd, $i-1 \equiv j$ cannot hold. Likewise, $i+1 \equiv j$ as well as $i \equiv j-1, j+1$ cannot occur.

In order to prove that $m$ is a multiple of $n$ we look at subsets $C$ of $\mathfrak{G}_{m}$ which are connected and on which $p\lceil C$ is injective. Such sets are called partial sections. If $p\lceil C$ is also surjective, in other words, if $\sharp C=n+1$ then $C$ is called section. We now prove

Claim 14 If $C$ is a partial section and $\sharp C>1$ then $C$ is contained in exactly one section.

To see this observe first that since $C$ is connected and $p$ is a $\triangleleft$-homomorphism, $p[C]$ is connected as well. Therefore, $C \subseteq\{0, \ldots, m\}$ is an interval as is $p[C] \subseteq\{0, \ldots, n\}$. If $i, i+1 \in C$ and $p: i \mapsto k$ then by the above claim $p: i+1 \mapsto k+1$ or $k-1$. If $p: i+1 \mapsto k+1$ then $p: i+2 \mapsto k+2$, for by the 
same argument $p: i+2 \mapsto k, k+2$ but $p(i+2)=k$ contradicts injectivity of $p\lceil C$. But if $p: i+1 \mapsto k-1$ then similarly $p: i+2 \mapsto k-2$. So, by induction, either $p\lceil C$ is a strictly increasing or strictly decreasing function. Now if $C=\{i, \ldots, j\}$ and $p[C]=\{k, \ldots, \ell\}$ and $p$ is monotone increasing then if $k>0, p(i)=k>0$ we get that by $( \pm c)$ either $i-1$ or $i+1$ is in the preimage of $k-1$. But $p(i+1)=k+1$ Thus $i>0$ and $p(i-1)=k-1$. So we add $i-1$ to $C$. Likewise, $p(j)=\ell$ and if $\ell<n$ then $j<m$ and we add $j+1$ to $C$. Similarly, if $p$ is decreasing.

Now $\mathfrak{G}_{m}$ contains exactly $m$ subsets $\{i, i+1\}$. $p$ is injective on each of them and they are all contained in one and only one section. Each section contains $n+1$ points and thus $n$ subsets $\{j, j+1\}$. Hence $n$ divides $m$ or $m=\omega$.

$(\Leftarrow)$ If $n=0$ take $p: m \mapsto 0$. If $n>0, \mathfrak{G}_{m}$ must be covered by sections in the following way. If $S, T$ are sections then $S=T$ or $\sharp(S \cap T) \leq 1$. Each section is an interval of $n+1$ points and each pair $\{i, i+1\}$ is in exactly one section. Hence the sections are $S_{k}=\{n k, \ldots, n(k+1)\}$. On each section $p$ is bijective. Suppose that $p$ is increasing on $S_{i}$. Then $p(n(k+1))=n$. Thus $p$ must be decreasing on $S_{i+1}$ and vice versa. Thus let $p$ be increasing on all even sections $S_{2 i}$ and decreasing on all odd sections $S_{2 i+1}$. Thus $p(i)=s$ iff $i=2 k n+s$ or $i=2(k+1) n-s$ for some $k$. We show that $p$ defined this way is a t-morphism. $(\triangleleft h)$ is as follows. We have $i \triangleleft i$ and $p(i) \triangleleft p(i)$. Moreover, if $i \triangleleft i+1$ or $i \triangleleft i-1$ then $i$ is odd. Now of $p(i)=s$ then either $i=2 k n+s$ or $i=(2 k+2) n-s$. In both cases $s$ is odd as well and $s \triangleleft s+1, s-1$ and $\{s-1, s+1\}=p[i-1, i+1]$. Similarly if $i$ is even. $(+c)$ and $(-c)$ are straightforward. If $i \in S, p(i) \triangleleft \ell$, then take $s=p^{-1}(\ell) \cap S$. Since $p\left\lceil S:\langle S, \triangleleft\rangle \rightarrow \mathfrak{G}_{n}\right.$ is an isomorphism, $i \triangleleft s$ as well. Similarly for $(-c)$.

With this result in our hands we can probe quite deeply into the structure of $\operatorname{Ext}(\mathbf{G a})$ and also prove the desired lemma. We have that $\mathbf{G a}=L \mathfrak{G}_{\omega}$ since $L \mathfrak{G}_{\omega} \supseteq L \mathfrak{G}_{n}$ for every $n$. Each logic containing Ga must be complete. This is due to

Theorem 15 Every extension of $\boldsymbol{K} t . \boldsymbol{A} \boldsymbol{t}_{n}^{+} . \boldsymbol{A} \boldsymbol{t}_{m}^{-}$is canonical.

Proof. The proof is a straightforward generalization of the one given in [1]. One has to observe that first of all $\mathbf{K} t$. $\mathbf{A l t}{ }_{n}^{+}$. $\mathbf{A l t} \mathbf{t}_{m}^{-}$is canonical and so if $\Lambda$ contains this logic, in every canonical generalized tense-frame frame $\langle\Gamma, \mathbb{G}\rangle$, $\mathbb{G} \subseteq 2^{G}, \Gamma$ is a frame for $\mathbf{K} t . \mathbf{A l t}_{n}^{+}$. $\mathbf{A l t}_{m}^{-}$and so for every point $g \in G$, $T^{k}(g, \Gamma)$ is finite. This is enough to see that $\Gamma$ must be a frame for $\Lambda$.

Incidentally this shows an interesting result on extensions of K4t. It is well known that an extension of $\mathbf{K} \mathbf{4}$ is tabular iff it is of finite codimension in $\operatorname{Ext}(\mathbf{K} 4)$ (see for example [10]). The above theorem shows that every 
extension of $\operatorname{Ext}(\mathbf{K} 4 t)$ of finite codimension is complete. (I owe this remark to Frank Wolter.)

Corollary 16 Every extension of $\boldsymbol{K} 4 t$ of finite codimension is complete and of finite alternativity.

Proof. If $\Lambda \supseteq \mathbf{K} 4 t$ is of finite codimension in $\operatorname{Ext}(\mathbf{K} 4 t)$ then $\Lambda_{+}$is of finite codimension in $\operatorname{Ext}(\mathbf{K} 4)$ and hence tabular. For if $\left\langle\Theta_{i} \mid i \in \alpha\right\rangle, \alpha \in \operatorname{Ord}$, is a strictly ascending chain of modal logics, then $\left\langle\Theta_{i}^{+} \mid i \in \alpha\right\rangle$ is a strictly ascending chain of tense logics by the fact that $(-)^{+}$is monotone and $\Theta_{i}=$ $\Theta_{j}$ implies $\Theta_{i}=\left(\Theta_{i}^{+}\right)_{+}=\left(\Theta_{j}^{+}\right)_{+}=\Theta_{j}$; thus $\alpha$ must be finite. Consequently, $\Lambda_{+}=L \Gamma$ for a frame $\Gamma$ with $\sharp G=n \in \omega$; thus K.Alt ${ }_{n} \subseteq \Lambda_{+}$from which $\mathbf{K}$.Alt ${ }_{1}^{+} \subseteq \Lambda$, by left adjointness. Similarly we prove that $\mathbf{K t . A l t}{ }_{m}^{-} \subseteq \Lambda$ for some $m \in \omega$. Thus $\Lambda$ is of finite alternativity and complete.

We do not believe, however, that every extension of finite codimension of either $\mathbf{K} 4 t$ or $\mathbf{S} 4 t$ is tabular although this is certainly true for $k$-transitive logics, for example $\mathbf{S 4 . 2} t$ and $\mathbf{S 4 . 3} t$ (see below). But now back to $\operatorname{Ext}(\mathbf{G a})$ ! The $\Pi$-irreducible elements are the $L \mathfrak{G}_{n}$ for $n \in \omega$. Every proper extension of $\mathbf{G a}$ which is not trivial is therefore an intersection $\Pi\left\langle L \mathfrak{G}_{n} \mid n \in F\right\rangle$ where $F \subseteq \omega$ is finite. For if $F$ is infinite we immediately have $\Pi\left\langle L \mathfrak{G}_{n}\right|$ $n \in F\rangle=\mathbf{G a}$ since $\mathfrak{G}_{\omega}$ is contained in $\prod_{U} G_{n}$ for a non-trivial ultrafilter $U$ on $F$. Equivalently, it is checked that the composition of the embedding $\mathfrak{G}_{n} \subseteq \mathfrak{G}_{\omega}$ with the t-morphism $\mathfrak{G}_{\omega} \rightarrow \mathfrak{G}_{m}$ is an $n$-localic map with respect to 0 . This has for consequence that every proper extension of $\mathbf{G a}$ is tabular while Ga itself is not. Such a logic is called pretabular.

Theorem 17 Ga is pretabular.

It is now straightforward to verify that the lattice of non-trivial proper extensions of $\mathbf{G a}$ is isomorphic to the distributive lattice freely $\sqcap$-generated by $\langle\omega, \mu\rangle$ with $m \mu n \Leftrightarrow n \mid m$ or $n=0$. Thus the upper part of $\operatorname{Ext}(\mathbf{G a})$ looks like this: 


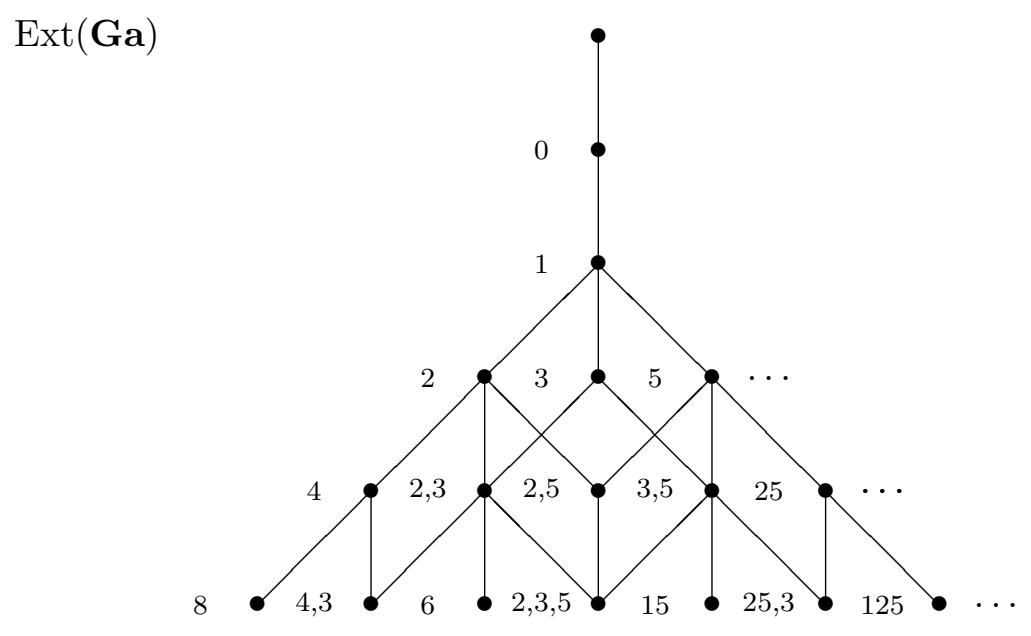

To the left of each node we have written numbers $n$ such that the node is the intersection of the logics of the corresponding $\mathfrak{G}_{n}$.

Proposition 18 There are infinitely many logics of codimension n in $\operatorname{Ext}(\boldsymbol{G a})$.

This proves a conjecture in [11] that $\mathbf{S} 4 t$ has infinitely many extensions of codimension 3 in contrast to $\mathbf{S} \mathbf{4}$ which for any $n$ has only finitely many. The proof of Lemma 11 is now easy. Clearly, bot $\Gamma_{0}$ and $\Gamma_{1}$ split the lattice. But for $n>1$ observe that the sequence $\left\langle\mathfrak{G}_{p}\right| p$ prime, $\left.p>n\right\rangle$ subreduces $\mathfrak{G}_{n}$. As we have noted, this implies also that none of the garlands $\mathfrak{G}_{n}$ split $\operatorname{Ext}(\mathbf{S} 4)$ unless $n \leq 1$. It will turn out soon that we cannot improve this result for $\operatorname{Ext}(\mathbf{S} 4 t)$. But for $\operatorname{Ext}(\mathbf{K} t)$ and $\operatorname{Ext}(\mathbf{K} 4 t)$ even these cases are ruled out. Look at the sequence $\left\langle\mathfrak{G}_{n}^{\circ} \mid n \in \omega\right\rangle$ where $\mathfrak{G}_{n}^{\circ}$ differs from $\mathfrak{G}_{n}$ in that $n \Varangle n$. The maps $p: \Gamma_{n}^{\circ} \leadsto \Gamma_{1}: j \mapsto j(\bmod 2)$ and $q: \Gamma_{n}^{\circ} \leadsto \Gamma_{0}: j \mapsto 0$ are $n$-localic with respect to 0 . Thus this sequence subreduces both frames in $\operatorname{Ext}(\mathbf{K} 4 t)$ and in $\operatorname{Ext}(\mathbf{K})$.

Lemma 19 For no $n, L \mathfrak{G}_{n}$ splits $\operatorname{Ext}(\boldsymbol{K} t), \operatorname{Ext}(\boldsymbol{K} 4 t)$.

It now remains to treat the clusters. Here the situation is quite similar to the situation of the garlands.

Lemma 20 Suppose $\Gamma$ is a cluster. Then $L \Gamma$ splits $\operatorname{Ext}(\boldsymbol{K} t)$ and $\operatorname{Ext}(\boldsymbol{K} 4 t)$ only if $\Gamma \cong \mathrm{x}$ and $\operatorname{Ext}(\boldsymbol{S} 4 t)$ only if $\Gamma \cong$ 
Proof. Let $n:=\sharp G>1$ and $\mathfrak{H}_{k}=\left\langle H_{k}, \triangleleft\right\rangle$ with $H_{k}=\{0, \ldots, k\} \times$ $\{1, \ldots, n\}-\{\langle k, n\rangle\}$ and $\langle i, j\rangle \triangleleft\left\langle i^{\prime}, j^{\prime}\right\rangle$ iff (i) $i$ is odd, $i^{\prime}=i+1$ or $i-1$ or (ii) $i$ is even and $i^{\prime}=i$. This can be visualized by

$\mathfrak{H}_{k}$

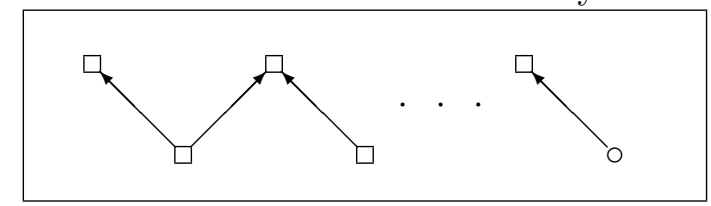

$\square$ denotes a cluster with $n$ points and $\circ$ a cluster with $n-1$ points. There is no t-morphism from $\mathfrak{H}_{k}$ into $\Gamma$ as there is no way to map a point belonging to an $n$-1-point cluster onto a $n$-point cluster.

Now look at $\mathbb{T}^{k}\left(\langle 0,0\rangle, \mathfrak{H}_{k}\right)$. Every point in $T^{k}\left(\langle 0,0\rangle, \mathfrak{H}_{k}\right)$ is contained in an $n$-point cluster since $\langle i, j\rangle \in T^{k}\left(\langle 0,0\rangle, \mathfrak{H}_{k}\right)$ iff $i<k$. Thus there is a t-morphism $p: \mathbb{T}^{k}\left(\langle 0,0\rangle, \mathfrak{H}_{k}\right) \rightarrow \Gamma$. Extend $p$ to a map $p^{+}: \mathfrak{H}_{k} \leadsto \Gamma$. $p^{+}$is $k$-localic with respect to $\langle 0, i\rangle$ for every $i$. Hence $L^{k}\left(\mathfrak{H}_{k}\right) \subseteq L^{k}(\Gamma)$. Consequently, the $\mathfrak{H}_{k}$ subreduce $\Gamma$.

Now we have collected all the material we need to prove the splitting theorems. Notice that a splitting frame for any of these logics can only be one-point cluster or a two-point garland. We will now show that the frames not excluded by the above lemmata are indeed splitting frames.

Theorem $21 \Lambda$ splits $\operatorname{Ext}(\boldsymbol{S} 4 t)$ iff $\Lambda=L \longmapsto \longrightarrow$ or $\Lambda=L \bullet$.

Proof. $(\Leftarrow)$ The nontrivial part is $\mathfrak{G}_{1}$. We will show that $\operatorname{Ext}(\mathbf{S} 4 t) / L \mathfrak{G}_{1}=$ S5t by proving that $(\dagger)$ of the Splitting Theorem holds for $m=1$. Therefore let $\mathfrak{A}$ be an algebra satisfying $L \mathfrak{A} \nsupseteq \mathbf{S} 5 t$. Then there is a set $C \in \mathbf{A}$ of $\mathfrak{A}$ such that $0<C \cap \tau^{+}-C$. Consequently, in the underlying Kripke-frame there are two points $s \triangleleft t$ such that $s \in C$ and $t \in \tau^{+}-C$ whence $t \nless s$. Now we have $\Delta\left(\mathfrak{G}_{1}\right)=\left(p_{a} \leftrightarrow \neg p_{b}\right) \wedge \diamond^{+} p_{b} \wedge \diamond^{-} p_{a} \wedge\left(\square^{+} p_{b} \leftrightarrow p_{b}\right) \wedge\left(\square^{-} p_{a} \leftrightarrow\right.$ $\left.p_{a}\right)$. Suppose that under these circumstances we can construct valuations $\alpha_{n}:\left\{p_{a}, p_{b}\right\} \rightarrow \mathbf{A}$ such that $s \in \alpha_{n}\left(p_{a} \wedge \square^{(n)} \Delta\left(\mathfrak{G}_{1}\right)\right)$. Then indeed (†) is satisfied and by the Splitting Theorem $\mathfrak{G}_{1}$ splits $\operatorname{Ext}(\mathbf{S} 4)$. To construct the $\alpha_{n}$ we define inductively subsets $A_{n}, B_{n}$ in $\mathfrak{A}$ as follows:

(0) $B_{0}=\tau^{+}-C$

$$
A_{0}=-B_{0}
$$

(i) $A_{2 k+1}=-B_{2 k+1}$

$$
B_{2 k+1}=B_{2 k} \cap A_{2 k}
$$

(ii) $A_{2 k+2}=A_{2 k+1} \cap B_{2 k+1}$

$B_{2 k+2}=-A_{2 k+1}$

Furthermore define $T_{0}=\{s\}, T_{2 k+1}=\boldsymbol{}^{+} T_{2 k}, T_{2 k+2}=\boldsymbol{\diamond}^{-} T_{2 k+1}$. The $T_{n}$ do not necessarily belong to A. Since $T_{2 k} \subseteq \tau^{-} T_{2 k}=\tau^{-} T_{2 k+1}$ 
and $T_{2 k+1} \subseteq \tau^{+}{ }^{-} T_{2 k+1}=\tau^{+} T_{2 k+1}$ it follows that $T_{n} \subseteq \tau^{(1)} T_{n+2}$. For $\tau^{(1)} T_{2 k+2}=\tau^{+} T_{2 k+2} \cap \tau^{-} T_{2 k+2} \supseteq T_{2 k+1} \cap \tau^{-} T_{2 k+1} \supseteq T_{2 k}$ and dually for odd $n$. Consequently, $s \in \tau^{(n)} T_{n+1}$.

We now verify the following claims:

(I) $\quad A_{n} \cap B_{n}=\emptyset$

$$
\begin{aligned}
& A_{n} \cup B_{n}=1 \\
& A_{n}=\tau^{-} A_{n} \\
& B_{n}=\tau^{+} B_{n} \\
& \text { (III) } \quad T_{n} \cap A_{n}=T_{n} \cap A_{n+1} \\
& T_{n} \cap B_{n}=T_{n} \cap B_{n+1} \\
& (I V) \quad T_{n} \subseteq \bullet^{+} B_{n} \cap{ }^{-} A_{n}
\end{aligned}
$$

(I) is trivial, (II-IV) are verified by induction; for (II) we only need to show $A \subseteq \tau^{-} A_{n}$ and $B \subseteq \tau^{+} B_{n}$. By symmetry of (i) and (ii) we may only take the case (i); $B_{2 k+1}=B_{2 k} \cap{ }^{-} A_{2 k}=\tau^{+} B_{2 k} \cap{ }^{-} A_{2 k} \subseteq$ $\tau^{+} B_{2 k} \cap \tau^{+} \checkmark^{-} A_{2 k}=\tau^{+}\left(B_{2 k} \cap{ }^{-} A_{2 k}\right)=\tau^{+} B_{2 k+1}, A_{2 k+1}=-B_{2 k+1}=$ $-\tau^{+} B_{2 k+1}={ }^{+} A_{2 k+1} \subseteq \tau^{-}{ }^{+} A_{2 k+1}=\tau^{-}{ }^{+} A_{2 k+1}=\tau^{-}-\tau^{+} B_{2 k+1}=$ $\tau^{-}-B_{2 k+1}=\tau^{-} A_{2 k+1}$. For (III) we now observe that $T_{2 k} \cap B_{2 k+1}=T_{2 k} \cap$ $B_{2 k} \cap{ }^{-} A_{2 k}=T_{2 k} \cap B_{2 k}$ since $T_{2 k} \subseteq \checkmark^{-} A_{2 k}$ by (IV). $T_{2 k} \cap A_{2 k+1}=T_{2 k} \cap A_{2 k}$ immediately follows. To prove (IV) we observe that if $t \in T_{2 k+1}$ there is a $s \in T_{2 k}$ such that $s \triangleright t$. By IH we have $s \in{ }^{+} B_{2 k}$ and therefore there is a $u \triangleright s$ such that $u \in T_{2 k} \cap B_{2 k}$. Now $u \in B_{2 k} \cap{ }^{-} A_{2 k}=B_{2 k+1}$ and, as $t \triangleleft s \triangleleft u$, $t \in{ }^{+} B_{2 k+1}$. To show $t \in{ }^{-} A_{2 k+1}$ we distinguish two cases: $(\alpha) t \in A_{2 k+1}$ and $(\beta) t \in B_{2 k+1}$. In case $(\alpha)$ we immediately have $t \in \checkmark^{-} A_{2 k+1}$ and in case $(\beta)$ we have $t \in \checkmark^{-} A_{2 k}$. But as $\checkmark^{-} A_{2 k+1}=\downarrow^{-}\left(A_{2 k} \cup \tau^{-} B_{2 k}\right) \supseteq \checkmark^{-} A_{2 k}$ we also have $t \in \diamond^{-} A_{2 k+1}$.

Now we put $\alpha: p_{a} \mapsto A_{n+1}, p_{b} \mapsto B_{n+1}$. It remains to be shown that $s \in$ $\alpha_{n}\left(p_{a} \wedge \square^{(n)} \Delta\left(\mathfrak{G}_{1}\right)\right)$. Notice that (I) - (IV) together yield $T_{n+1} \subseteq \alpha_{n}\left(\Delta\left(\mathfrak{G}_{1}\right)\right)$ whence $\{s\} \subseteq \tau^{(n)} T_{n+1} \subseteq \alpha_{n}\left(\square^{(n)} \Delta\left(\mathfrak{G}_{1}\right)\right)$. And since by (III) and the fact that $s \in T_{n+1}$ we have $s \in \alpha_{n}\left(p_{a}\right)$, everything is proved.

From this theorem one can deduce that the lattice $\operatorname{Ext}(\mathbf{S} 4 t)$ has exactly two elements of codimension 2. For if $\Lambda \nsubseteq L \bullet \longrightarrow$ then $\Lambda \supseteq$ S5t; thus if $\Lambda$ is of codimension 2 it is the logic of the two point cluster.

Theorem $22 \Theta$ splits $\operatorname{Ext}(\boldsymbol{K} 4 t)$ and $\operatorname{Ext}(\boldsymbol{K} t)$ iff $\Theta=L \overline{\mathrm{x}}$.

Proof. $\mathrm{X}$ is cycle-free and therefore splits $\operatorname{Ext}(\mathbf{K} t)$. A fortiori it splits $\operatorname{Ext}(\mathbf{K} 4 t)$.

As we see, compared to modal logics tense logics is a lost paradise. By way of consolation I prove some 'positive' results:

Proposition 23 LA splits $\operatorname{Ext}(\boldsymbol{S} 4.2 t)(\operatorname{Ext}(\boldsymbol{S} 4.3 t), \operatorname{Ext}(\boldsymbol{S} 5 t))$ iff $\mathfrak{A}$ is f.s.i. 
Moreover, an extension of these lattices is tabular iff it is of finite codimension.

Proof. We will show that $\mathbf{S 4 . 2} t$ is 2 -transitive. Then since $\mathbf{S 4 . 2} t \subset$ $\mathbf{S} 4.3 t \subset \mathbf{S} 5 t$ all logics are 2-transitive. Since they have f.m.p. (see Burgess $[84])$ the proposition is proved. Now $\mathbf{S} 4.2 t=\mathbf{S} 4 t\left(\diamond^{+} \square^{+} p \rightarrow \square^{+} \diamond^{+} p\right)$. Thus $\diamond^{-} \diamond^{+} p \vdash \diamond^{-} \diamond^{+} \square^{+} \diamond^{-} p \vdash \diamond^{-} \square^{+} \diamond^{+} \diamond^{-} p \vdash \diamond^{+} \diamond^{-} p$. By the laws of S4t, 2transitivity is equivalent to showing $\diamond^{+} \diamond^{-} \diamond^{+} p \vee \diamond^{-} \diamond^{+} \diamond^{-} p \rightarrow \diamond^{+} \diamond^{-} p \vee$ $\diamond^{-} \diamond^{+} p$. But this holds because $\diamond^{+} \diamond^{-} \diamond^{+} p \vee \diamond^{-} \diamond^{+} \diamond^{-} p \vdash \diamond^{+} \diamond^{+} \diamond^{-} p \vee$ $\diamond^{+} \diamond^{-} \diamond^{-} p \vdash \diamond^{+} \diamond^{-} p \vee \diamond^{-} \diamond^{+} p$. Now take an extension $\Lambda$ of finite codimension; then it is complete and, say, of alternativity $n$. So $\Lambda=L \Gamma$ for some frame $\Gamma$. By 2-transitivity, $\mathbb{T}^{2}(g, \Gamma)$ is a connected component of $\Gamma$ for any $g$ and by $n$-alternativity $\sharp \mathbb{T}^{2}(g, \Gamma) \leq 2 n^{2}$. As $\Lambda=\prod\left\langle L \mathbb{T}^{2}(g, \Gamma) \mid g \in \Gamma\right\rangle$ is of finite codimension, only finitely many of the logics $L \mathbb{T}^{2}(g, \Gamma)$ can be different, so we can assume that $\Gamma$ contains only finitely many generated subframes. But then $\Gamma$ is finite. 


\section{References}

[1] Fabio Bellissima. On the lattice of extensions of the modal logics K.Alt ${ }_{n}$. Archive for Mathematical Logic, 27:107-114, 1988.

[2] W. J. Blok. On the degree of incompleteness of modal logics. Bulletin of the Section of Logic of the Polish Academy of Sciences, 7:167 - 175, 1978.

[3] Wim Blok. On the degree of incompleteness in modal logics and the covering relation in the lattice of modal logics. Technical Report 78-07, Department of Mathematics, University of Amsterdam, 1980.

[4] Wim Blok and Don Pigozzi. On the structure of varieties with equationally definable principal congruences, I. Algebra Universalis, 15:195 $-227,1982$.

[5] John P. Burgess. Basic tense logic. In Dov M. Gabbay and Franz Guenthner, editors, Handbook of Philosophical Logic, volume 2, pages 89-133. Reidel, 1984.

[6] G. Kalmbach. Orthomodular Lattices. Academic Press, London, 1983.

[7] Marcus Kracht. An almost general splitting theorem for modal logic. Studia Logica, 49:455-470, 1990.

[8] Ralph McKenzie. Equational bases and nonmodular lattice varieties. Transactions of the American Mathematical Society, 174:1 - 43, 1972.

[9] Wolfgang Rautenberg. Der Verband der normalen verzweigten Modallogiken. Mathematische Zeitschrift, 156:123-140, 1977.

[10] Wolfgang Rautenberg. Klassische und nichtklassische Aussagenlogik. Vieweg, Braunschweig/Wiesbaden, 1979.

[11] Wolfgang Rautenberg. More about the lattice of tense logics. Bull. of the Section of Logic of the Polish Academy of Sciences, 8:21 - 29, 1979.

[12] Wolfgang Rautenberg. Splitting lattices of logics. Archiv für Mathematische Logik, 20:155-159, 1980.

[13] S. K. Thomason. Semantic analysis of tense logic. Journal of Symbolic Logic, 37:150-158, 1972. 